\title{
Prevalência de Fatores de Risco Cardiovascular em Funcionários de uma Instituição de Ensino Superior
}

\author{
Prevalence of Cardiovascular Risk Factors in \\ employees of Brazilian University
}

\author{
Maristela Sanches BERTASSO-BORGES*, \\ Daniela Costa PRATES, Andreia Fernandes \\ Marcondes Godoy SILVA, Ana Paula Fernandes PEZZINI \\ Centro Universitário de Rio Preto, UNIRP. Rua Ivete Gabriel Atique, 45. \\ Boa Vista, São José do Rio Preto, SP, Brasil. \\ E-mail: bertasso@unirp.edu.br
}

\begin{abstract}
Cardiovascular diseases have been the main cause of death in Brazil and the identification of cardiovascular risk factors is crucial to effective prevention. This study aimed to determine the prevalence of cardiovascular risk factors in employees of a University in São José do Rio Preto. This analysis was done by a study transverse and descriptive carried out from a questionnaire for the identification of cardiovascular risk factors, anthropometric measures and arterial blood pressure in employees. A total of 127 employees were assessed, being 84 (66.14\%) of them females. The age group most prevalent is 21 to 30 years (37\%). Arterial hypertension was identified in $7.09 \%$ of the people and $17.32 \%$ of them were in the pre-hypertension range. Positive family history was reported by $82.68 \%$ of the employees. Approximately $50 \%$ of the population reported alcoholism, with prevalence in males $(69.77 \%)$. The frequency of a sedentary lifestyle was high in females (73.81\%) and 52.76\% of the total population was overweight. For females there was statistical significance for waist circumference in the analysis by age groups. Based on the results we conclude that hypertension, family history, alcoholism, overweight and a sedentary lifestyle are the main cardiovascular risk factors in this population of employees.
\end{abstract}

KEYWORDS: Cardiovascular diseases; risk factors; lifestyle; epidemiology; Brazil.

RESUMO

As doenças cardiovasculares tem sido a principal causa de morte no Brasil e a identificação dos fatores de risco cardiovascular é crucial para uma prevenção efetiva. Este trabalho teve como objetivo determinar a prevalência de fatores de risco cardiovascular em funcionários de uma Instituição de Ensino Superior de São José do Rio Preto. Foi feito um estudo transversal e descritivo realizado a partir de um questionário para identificação de fatores de risco cardiovascular, medidas antropométricas e de pressão arterial em funcionários. Avaliaram-se 127 funcionários, sendo 84 (66,14\%) do sexo feminino. A faixa etária mais prevalente é a de 21 a 30 anos (37\%). Hipertensão arterial foi identificada em 7,09\% dos indivíduos e 17,32\% estavam na faixa de pré-hipertensão. História familiar positiva foi relatada por $82,68 \%$ dos funcionários. Aproximadamente $50 \%$ da população relataram etilismo, com prevalência no sexo masculino (69,77\%). A frequência de sedentarismo foi elevada no sexo feminino $(73,81 \%)$ e $52,76 \%$ da população total encontram-se nas faixas de sobrepeso. Para o sexo feminino houve significância estatística para circunferência de cintura na análise por faixas etárias. Com base nos resultados pôde-se concluir que hipertensão, história familiar, etilismo, sobrepeso e sedentarismo são os principais fatores de risco cardiovascular nesta população de funcionários.

PALAVRAS CHAVE: Doenças cardiovasculares, fatores de risco, estilo de vida, epidemiologia, Brasil. 


\section{LISTA DE ABREVIAÇÕES:}

$\begin{array}{ll}\text { DCV } & \text { Doenças Cardiovasculares } \\ \text { IMC } & \text { Índice de Massa Corpórea } \\ \text { CC } & \text { Circunferência de Cintura } \\ \text { RCQ } & \text { Razão Circunferência da Cintura e Quadril } \\ \text { PA } & \text { Pressão Arterial } \\ \text { HAS } & \text { Hipertensão Arterial Sistêmica } \\ \text { CQ } & \text { Circunferência de Quadril } \\ \text { SUS } & \text { Sistema Único de Saúde }\end{array}$

\section{INTRODUÇÃO}

No Brasil, as doenças cardiovasculares tem sido a principal causa de morte e são responsáveis por alta frequência de internações, ocasionando custos médicos e socioeconômicos elevados. Como exemplo, em 2007, foram a causa de 1.157.509 internações registradas no Sistema Único de Saúde (SUS) (1) As doenças cardiovasculares (DCV) são a primeira causa de morte no Brasil, sendo responsáveis, em 2008, por $34 \%$ dos óbitos da população adulta e por $40,8 \%$ dos óbitos de indivíduos com 60 anos ou mais. Entre seus principais subgrupos, estão as doenças cerebrovasculares e as doenças isquêmicas do coração, que totalizaram mais de $60 \%$ dos óbitos por DCV (2). A cardiologia passa por um processo gradual de transição entre a conduta intervencionista e a preventiva. A expressão fatores de risco refere-se às características que certas pessoas podem desenvolver e que se relacionam ou não ao aparecimento de doenças coronarianas. Os principais fatores de risco cardiovasculares são: hipertensão arterial, dislipidemia, diabetes mellitus, obesidade, sedentarismo, predisposição genética, sexo, etnia e alcoolismo. Numerosos estudos associam o tabagismo à maior morbidade e mortalidade por doença coronariana aterosclerótica. Esta associação advém dos múltiplos efeitos deletérios causados pelo tabagismo nos mecanismos vasomotor, de aterogênese, e arritmogênico (3). Diversos levantamentos epidemiológicos constataram a elevada presença de fatores de risco na população brasileira, mas com padrões regionais distintos. A identificação e quantificação dos riscos tem sido os principais instrumentos utilizados pela medicina preventiva para nortear ações que tragam diminuição da morbimortalidade e aumento na melhoria da qualidade de vida. As síndromes coronarianas são responsáveis por $32 \%$ dos óbitos no Brasil, sendo a terceira maior causa de custos aos cofres públicos. Dentre estas síndromes, o infarto agudo do miocárdio é a principal cardiopatia isquêmica, apresentando elevados índices de mortalidade (4). Um evento coronariano agudo é a primeira manifestação da doença aterosclerótica em pelo menos meta- de dos indivíduos que apresentam essa complicação. Desta forma, a identificação dos indivíduos assintomáticos, mais predispostos, é crucial para a prevenção efetiva. A estimativa do risco de doença aterosclerótica resulta do somatório do risco causado por cada um dos fatores mais a potenciação causada por sinergismos entre alguns desses fatores (5).Uma parcela significativa da população brasileira desconhece a importância de se realizar o controle dos fatores de risco, elemento central para se prevenir as complicações das doenças cardiovasculares. O objetivo deste trabalho foi determinar a prevalência de fatores de risco para doença cardiovascular em funcionários de uma Instituição de Ensino Superior da cidade de São José do Rio Preto, SP.

\section{MÉTODOS}

Estudo transversal e descritivo, realizado no período entre março e outubro de 2010, no qual funcionários de uma Instituição de Ensino Superior de São José do Rio Preto, SP foram investigados sobre prevalência de fatores de risco para doença cardiovascular, através de um questionário. O projeto foi aprovado pelo Comitê de Ética em Pesquisa do Instituto de Moléstias Cardiovasculares de São José do Rio Preto (CEP-IMC 001-015-2009). Antes da aplicação do questionário, os participantes foram informados sobre o objetivo do estudo e os procedimentos, e assinaram o Termo de Consentimento Livre e Esclarecido. Os critérios de inclusão adotados foram: adultos (20-59 anos), ligados profissionalmente à Instituição, não exercendo a função docente. Como critério de exclusão adotou-se: entrega do questionário em branco ou parcialmente respondido, e falta de assinatura no termo de consentimento informado.

O questionário era composto por oito itens, iniciando com idade, sexo, grau de escolaridade, tabagismo, consumo de álcool e realização de atividade física regular. No item histórico pessoal foi perguntado se o indivíduo já havia feito tratamento para hipertensão arterial sistêmica, hipercolesterolemia e diabetes mellitus, ou tinha recebido algum destes diagnósticos por um médico. Foram identificados como portadores de tais doenças, os funcionários que, no momento da entrevista, relataram uso de medicamentos hipoglicemiantes, ou para tratamento de hipercolesterolemia ou hipertrigliceridemia, assim como medicamentos anti-hipertensivos. Em histórico médico familiar foi perguntado sobre a existência de familiares de primeiro grau com história de risco cardiovascular.

No item composição corporal foram avaliadas medidas de peso e altura, utilizadas para se calcular 
o Índice de Massa Corpórea (IMC). O IMC foi obtido por meio do quociente: IMC = peso (kilograma) / altura $^{2}$ (metros) para a classificação do estado nutricional, segundo a Organização Mundial de Saúde (6). Foram considerados normais valores de IMC entre $18,50 \mathrm{~kg} / \mathrm{m}^{2}$ e $24,99 \mathrm{~kg} / \mathrm{m}^{2}$; sobrepeso quando o IMC se encontrava entre $25 \mathrm{~kg} / \mathrm{m}^{2}$ e $29,99 \mathrm{~kg} / \mathrm{m}^{2}$ e obesidade quando IMC $\geq 30 \mathrm{~kg} / \mathrm{m}^{2}$. A circunferência de cintura (CC) e a razão entre as medidas da circunferência da cintura e quadril (RCQ) foram estudadas como indicador de obesidade abdominal. A medida da CC foi obtida por meio de fita métrica inextensível, no ponto médio entre a crista ilíaca e a borda inferior da última costela. Foram definidos como valores de risco cardiovascular a CC acima de $80 \mathrm{~cm}$ para as mulheres e acima de 94 para os homens. A relação cintura-quadril foi obtida a partir dos valores de circunferência da cintura e do quadril e para a classificação destes foram utilizados os pontos de corte recomendados pela Organização Mundial de Saúde (6). A circunferência do quadril foi aferida em centímetros na área de maior protuberância glútea, em um plano horizontal. A relação cintura-quadril foi obtida a partir dos valores de circunferência da cintura e do quadril e para a classificação destes foram utilizados os pontos de corte recomendados pela Organização Mundial de Saúde (6). A pressão arterial (PA) foi obtida segundo procedimentos específicos (Esfigmomanômetro marca BIC, modelo ML 040/2003). Níveis de pressão arterial foram verificados e classificados de acordo com as VI Diretrizes Brasileiras de Hipertensão (1). No último item, estresse, questionou-se se o participante se considerava tranquilo ou estressado diante das atividades cotidianas.

\section{Análise Estatística}

Para determinar a significância estatística foi utilizado o teste Exato de Fisher e a análise de variância (Anova) através do programa estatístico EPIINFO versão 6,0. O nível de significância adotado foi de $5 \%$.

\section{RESULTADOS}

Foram avaliados 127 funcionários, sendo 84 $(66,14 \%)$ do sexo feminino e $43(33,86 \%)$ do sexo masculino. A faixa etária mais prevalente foi a de 21 a 30 anos (37\%), tanto para homens quanto para mulheres. Em relação ao grau de instrução, foi verificado que inexistiam analfabetos e que a maioria possuía grau de instrução igual ou superior ao ensino médio completo $(85,1 \%)$, conforme mostrado na Tabela 1. Os dados sobre prevalência e distribuição por sexo de pressão arterial sistêmica, diabetes, colesterolemia, história familiar, tabagismo, sedentarismo, etilismo e estresse na população de funcionários estudada encontram-se sumarizados na Tabela 2. Em relação à pressão arterial, foi verificado que $75,59 \%$ da população apresentavam níveis pressóricos considerados normais ou desejáveis. Uma associação altamente significativa foi observada entre sexo feminino e níveis normais de pressão arterial $(p=0,0004)$. Os pré-hipertensos atingiram $17,32 \%$ da população, e a Hipertensão Arterial Sistêmica (HAS) (estágio 1 ou 2) foi identificada em apenas 9 indivíduos, correspondendo a $7,09 \%$ da população estudada, com uma associação significativa entre o sexo masculino e hipertensão nível $\mathrm{I}(\mathrm{p}=0,0061)$. No que diz respeito à intolerância a glicose, apenas $3,15 \%$ dos funcionários relataram diabetes. A hipercolesterolemia foi apontada por 9,45\% dos indivíduos (Tabela 2).

História familiar positiva prévia de doenças cardiovasculares como ataque cardíaco, hipertensão, diabetes, acidente cerebral, hipercolesterolemia, cirurgias cardíacas e obesidade entre parentes de primeiro grau foi relatada pela maioria dos funcionários $(82,68 \%)$, sendo 70 mulheres, correspondendo a $83,33 \%$ da população feminina, e 35 homens $(81,4 \%)$ (Tabela 2$)$.

O tabagismo foi encontrado como hábito em 13 $(10,24 \%)$ dos funcionários, sendo $9(10,71 \%)$ do sexo feminino e $4(9,30 \%)$ do sexo masculino sem diferença significativa, entre os sexos $(P=1,0000)$. No sexo feminino, 7,14\% das mulheres relataram tabagismo pregresso, e com relação aos homens $16,28 \%$ enquadraram-se nesta situação, indicando que, no total, $21,26 \%$ da população estudada está ou esteve exposta a esse fator de risco (Tabela 2).

No item atividade física, constatou-se elevado nível de sedentarismo (84 funcionários, 66,14\%), com frequência maior no sexo feminino $(73,81 \%)$. Pode ser observada uma associação significativa entre sexo feminino e sedentarismo $(\mathrm{p}=0,0168)$ (Tabela 2$)$.

Aproximadamente $50 \%$ da população relataram etilismo, com prevalência no sexo masculino $(69,77 \%)$, resultando em uma associação altamente significativa entre sexo masculino e consumo de álcool $(\mathrm{p}=0,0014)$. Quanto ao fator estresse, a maioria das pessoas relatou sentirem-se tranquilas, e 33,86\% dos indivíduos relataram estresse. Destes, a maior frequência está relacionada ao sexo feminino $(38,10 \%)$, porém sem diferença significativa (Tabaela 2). 
Tabela 1 - Dados demográficos relativos à faixa etária e ao grau de instrução da população estudada, de acordo com o sexo.

\begin{tabular}{|c|c|c|c|c|c|c|}
\hline \multirow{2}{*}{ Faixa Etária } & \multicolumn{2}{|c|}{ Sexo Feminino } & \multicolumn{2}{|c|}{ Sexo Masculino } & \multicolumn{2}{|c|}{ Total } \\
\hline & $\mathrm{N}^{\circ}$ & $\%$ & $\mathrm{~N}^{\circ}$ & $\%$ & $\mathrm{~N}^{\circ}$ & $\%$ \\
\hline $18-20$ & 2 & 2,4 & 1 & 2,3 & 3 & 2,4 \\
\hline $21-30$ & 29 & 34,5 & 18 & 41,9 & 47 & 37,0 \\
\hline $31-40$ & 25 & 29,8 & 13 & 30,2 & 38 & 29,9 \\
\hline $41-50$ & 17 & 20,2 & 6 & 14 & 23 & 18,1 \\
\hline $51-60$ & 8 & 9,5 & 3 & 7 & 11 & 8,7 \\
\hline $61-70$ & 2 & 2,4 & 2 & 4,6 & 4 & 3,1 \\
\hline ignorado & 1 & 1,2 & 0 & 0 & 1 & 0,8 \\
\hline Total & 84 & 100 & 43 & 100 & 127 & 100 \\
\hline \multirow{2}{*}{ Escolaridade } & \multicolumn{2}{|c|}{ Sexo Feminino } & \multicolumn{2}{|c|}{ Sexo Masculino } & \multicolumn{2}{|c|}{ Total } \\
\hline & $\mathrm{N}^{\circ}$ & $\%$ & $\mathrm{~N}^{\circ}$ & $\%$ & $\mathbf{N}^{\circ}$ & $\%$ \\
\hline Fundamental incompleto & 4 & 4,8 & 3 & 7 & 7 & 5,5 \\
\hline Fundamental completo & 4 & 4,8 & 2 & 4,7 & 6 & 4,7 \\
\hline Médio incompleto & 3 & 3,6 & 3 & 7 & 6 & 4,7 \\
\hline Médio completo & 22 & 26,2 & 3 & 7 & 25 & 19,7 \\
\hline Superior incompleto & 18 & 21,4 & 15 & 34,8 & 33 & 26 \\
\hline Superior completo & 33 & 39,2 & 17 & 39,5 & 50 & 39,4 \\
\hline Total & 84 & 100 & 43 & 100 & 127 & 100 \\
\hline
\end{tabular}

Tabela 2 - Prevalência e distribuição por sexo de pressão arterial sistêmica, diabetes, colesterolemia, história familiar, tabagismo, sedentarismo, etilismo e estresse na população de funcionários estudada.

\begin{tabular}{|c|c|c|c|c|c|c|c|}
\hline \multirow[t]{2}{*}{ Sexo } & \multicolumn{2}{|c|}{$\begin{array}{c}\text { Feminino } \\
(n=84)\end{array}$} & \multicolumn{2}{|c|}{$\begin{array}{l}\text { Masculino } \\
(n=43)\end{array}$} & \multicolumn{2}{|c|}{$\begin{array}{c}\text { Total } \\
(\mathrm{n}=127)\end{array}$} & \multirow[t]{2}{*}{$P^{*}$} \\
\hline & $\mathbf{N}$ & $\%$ & $\mathbf{N}$ & $\%$ & $\mathbf{N}$ & $\%$ & \\
\hline \multicolumn{8}{|l|}{ Classe de PA } \\
\hline Normal $(\leq 120 \times 80 \mathrm{mmH})$ & 72 & 85,71 & 24 & 55,82 & 96 & 75,59 & 0,0004 \\
\hline Pré-HÁ (120-139x80-89) & 11 & 13,10 & 11 & 25,58 & 22 & 17,32 & 0,0883 \\
\hline Estágio 1 (140-159×90-99) & 1 & 1,19 & 6 & 13,95 & 7 & 5,51 & 0,0061 \\
\hline Estágio 2 (160-179×100-109) & 0 & 0 & 2 & 4,65 & 2 & 1,58 & 0,1129 \\
\hline \multicolumn{8}{|l|}{ Diabetes } \\
\hline Sim & 3 & 3,57 & 1 & 2,33 & 4 & 3,15 & 1,0000 \\
\hline Não & 81 & 96,43 & 42 & 97,67 & 123 & 96,85 & \\
\hline \multicolumn{8}{|l|}{ Colesterol } \\
\hline Sim & 9 & 10,72 & 3 & 6,98 & 12 & 9,45 & 0,5470 \\
\hline Não & 75 & 89,28 & 40 & 93,02 & 115 & 90,55 & \\
\hline \multicolumn{8}{|l|}{ História familiar } \\
\hline Positiva & 70 & 83,33 & 35 & 81,40 & 105 & 82,68 & 0,8074 \\
\hline Negativa & 14 & 16,67 & 8 & 18,60 & 22 & 17,32 & \\
\hline \multicolumn{8}{|l|}{ História familiar } \\
\hline Sim & 9 & 10,71 & 4 & 9,30 & 13 & 10,24 & 1,0000 \\
\hline Não & 75 & 89,29 & 39 & 90,69 & 114 & 89,76 & \\
\hline \multicolumn{8}{|l|}{ Sedentarismo } \\
\hline Positivo & 62 & 73,81 & 22 & 51,16 & 84 & 66,14 & 0,0168 \\
\hline Negativo & 22 & 26,19 & 21 & 48,84 & 43 & 33,86 & \\
\hline
\end{tabular}




\begin{tabular}{|c|c|c|c|c|c|c|c|}
\hline \multirow{2}{*}{ Sexo } & \multicolumn{2}{|c|}{$\begin{array}{c}\text { Feminino } \\
(n=84)\end{array}$} & \multicolumn{2}{|c|}{$\begin{array}{c}\text { Masculino } \\
(n=43)\end{array}$} & \multicolumn{2}{|c|}{$\begin{array}{c}\text { Total } \\
(n=127)\end{array}$} & \multirow{2}{*}{$P *$} \\
\hline & $\mathbf{N}$ & $\%$ & $\mathbf{N}$ & $\%$ & $\mathbf{N}$ & $\%$ & \\
\hline \multicolumn{8}{|l|}{ Estilismo } \\
\hline Positivo & 33 & 39,29 & 30 & 69,77 & 63 & 49,61 & 0,0014 \\
\hline Negativo & 51 & 60,71 & 13 & 30,23 & 64 & 50,39 & \\
\hline \multicolumn{8}{|l|}{ Estresse } \\
\hline Positivo & 32 & 38,10 & 11 & 25,58 & 43 & 33,86 & 0,1720 \\
\hline Negativo & 52 & 61,90 & 32 & 74,42 & 84 & 66,14 & \\
\hline
\end{tabular}

* Exato de Fisher

Com relação ao índice de massa corpórea (IMC), $52,76 \%$ da população total encontrou-se nas faixas de pré-obesidade ou obesidade. Houve variação entre os dois sexos, sendo que para o sexo feminino, a maior parte da população estudada $(52,38 \%)$ encontrava-se na faixa de normalidade. No sexo masculino, a maior prevalência foi de pré-obesidade $(41,86)$. Uma associação significativa foi observada entre IMC normal e sexo feminino $(p=0,0395)$. Obesidade grave (IMC $\geq 40$ ) foi encontrada em 4 funcionários $(3,15 \%)$, de ambos os sexos.

Não houve diferença estatisticamente significativa quanto ao sobrepeso e obesidade entre os dois sexos (Tabela 3)A Tabela 4 apresenta a distribuição do índice de massa corporal (IMC) expresso por média e desvio padrão, de acordo com sexo e grau de instrução. O IMC dos participantes variou de $18,0 \mathrm{~kg} / \mathrm{m}^{2}$ a $42,59 \mathrm{~kg} / \mathrm{m}^{2}$, com média de $25,30 \pm$ 5,17 para o sexo feminino e $27,74 \pm 5,25$ para o sexo masculino. Quando foi comparada a variância dentro de cada nível educacional para o sexo masculino e feminino observou-se uma variação significativa no sexo masculino que apresentou maiores valores de IMC no grupo com grau de instrução maior (ANOVA $\mathrm{P}=0,015$ ). Para o sexo masculino não houve significância estatística quando se analisou a circunferência de cintura (CC), circunferência de quadril (CQ) e relação circunferência cintura/circunferência do quadril (RCQ) por faixa etária. A Tabela 5 apresenta as médias e desvios padrão destes parâmetros. Pode ser observado um aumento destes valores na faixa etária de 41-50 anos, porém, sem significância estatística. A Tabela 6 sumariza os dados das distribuições destas mesmas variáveis para o sexo feminino. Todas as variáveis apresentaram aumento com o avançar da idade, com significância estatística apenas para $C C(p=0,003)$, na análise por faixas etárias. A CC apresentou incremento significativo com o aumento da idade, com valores que variaram de 76,42 $\pm 10,71$ na faixa com menos de 30 anos, para $92,70 \pm$ 16,02 após os 50 anos de idade, com uma associação altamente significativa entre a primeira faixa etária e a terceira assim como entre a primeira e a quarta faixa etária.

Tabela 3 - Distribuição do índice de massa corporal (IMC) por sexo, de acordo com a classificação da Organização Mundial da Saúde.

\begin{tabular}{|l|c|c|c|c|c|c|c|}
\hline \multicolumn{1}{|c|}{ Classificação IMC (OMS) } & \multicolumn{2}{|c|}{ Sexo Feminino } & Sexo Masculino & \multicolumn{3}{c|}{ Total } \\
\hline & $\mathbf{n}$ & $\%$ & $\mathbf{N}$ & $\%$ & $\mathbf{n}$ & $\%$ \\
\hline Baixo peso (até 18,4) & 2 & 2,38 & 0 & 0 & 2 & 1,57 & 0,5486 \\
\hline Normalidade (18,5 a 24,9) & 44 & 52,38 & 14 & 32,56 & 58 & 45,67 & 0,0395 \\
\hline Pré-obeso (25,0 a 29,9) & 25 & 29,76 & 18 & 41,86 & 43 & 33,86 & 0,2343 \\
\hline Obesidade Classe I (30,0 a 34,9) & 10 & 11,91 & 8 & 18,60 & 18 & 14,17 & 0,4201 \\
\hline Obesidade Classe II (35,0 a 39,9) & 1 & 1,19 & 1 & 2,33 & 2 & 1,58 & 1,0000 \\
\hline Obesidade Classe III ( $\geq 40)$ & 2 & 2,38 & 2 & 4,65 & 4 & 3,15 & 0,6035 \\
\hline Total & 84 & 100 & 43 & 100 & 127 & 100 & \\
\hline
\end{tabular}

* Exato de Fisher 
Tabela 4 - Distribuição do índice de massa corporal (IMC) $\left(\mathrm{kg} / \mathrm{m}^{2}\right)$ expresso por média e desvio padrão, de acordo com sexo e grau de instrução.

\begin{tabular}{|c|c|c|c|}
\hline Grau de instrução & Sexo Feminino & Sexo Masculino & ANOVA p \\
\hline Fundamental incompleto & $30,98 \pm 11,01$ & $30,17 \pm 2,99$ & 0,9037 \\
\hline Fundamental completo & $28,02 \pm 4,52$ & $26,40 \pm 6,36$ & 0,7266 \\
\hline Médio incompleto & $24,03 \pm 3,80$ & $26,53 \pm 9,24$ & 0,6864 \\
\hline Médio completo & $25,44 \pm 3,91$ & $28,22 \pm 1,62$ & 0,2407 \\
\hline Superior incompleto & $25,44 \pm 4,21$ & $27,17 \pm 6,27$ & 0,6351 \\
\hline Superior completo & $24,27 \pm 5,44$ & $28,10 \pm 4,62$ & $0,015^{*}$ \\
\hline Total & $25,30 \pm 5,17$ & $27,74 \pm 5,25$ & \\
\hline
\end{tabular}

* ANOVA: pós teste com Teste $\mathrm{t}: \mathrm{p}=0,0163$

\section{DISCUSSÃO}

O presente estudo busca contribuir para o conhecimento acerca da prevalência de fatores de risco para doenças cardiovasculares em funcionários de uma Instituição de Ensino Superior de São José do Rio Preto e, assim, poder alertar os servidores e a comunidade acadêmica - estudantes, educadores e gestores de educação - para a importância da elaboração de programas de prevenção, incentivando a adoção de hábitos de vida saudáveis.

Um estudo sobre mortalidade por doenças cardiovasculares (DCV) e níveis socioeconômicos na população de São José do Rio Preto, estado de São Paulo mostrou que o coeficiente de mortalidade por DCV, em São José do Rio Preto, apresenta grandes valores para a população acima de 50 anos e é maior para os residentes na área com os piores níveis socioeconômicos. No ano de 2003, em São José do Rio Preto, a mortalidade proporcional por DCV, foi igual a $31,7 \%$ e o coeficiente de mortalidade por estas doenças atingiu o valor de 33,9\% maior para os homens. As três principais causas dentro desse grupo foram: a doença cérebro vascular (53,6 óbitos por 100.000 habitantes), o infarto agudo do miocárdio e a doença hipertensiva (7).

A hipertensão arterial sistêmica (HAS) é uma condição clínica multifatorial caracterizada por níveis elevados e sustentados de pressão arterial (PA). Associa-se frequentemente a alterações funcionais e/ ou estruturais dos órgãos-alvo (coração, encéfalo, rins e vasos sanguíneos) e a alterações metabólicas, com consequente aumento do risco de eventos cardiovasculares fatais e não fatais (1). No presente estudo, 75,59\% da população apresentavam níveis pressóricos considerados normais ou desejáveis. Uma associação altamente significativa foi observada entre sexo feminino e níveis normais de pressão arterial $(\mathrm{p}=0,0004)$. Por outro lado, $24,41 \%$ dos indivíduos analisados apresentavam pré-hipertensão ou hipertensão, o que está de acordo com os dados encon- trados em uma amostra de 1.717 indivíduos representativa da população adulta e urbana da cidade de São José do Rio Preto - SP/Brasil, no qual a prevalência de hipertensão foi de 25,2\% (8).

No presente trabalho, os pré-hipertensos atingiram $17,32 \%$ da população, e a HAS (estágio 1 ou 2) foi identificada em $7,09 \%$ da população estudada. Diferentemente, um estudo de prevalência de risco cardiovascular em uma população de industriários no Brasil demonstrou que apenas $27 \%$ da população apresentavam níveis pressóricos considerados normais ou desejáveis, pré-hipertensos atingiram $45 \%$ da população, e a HAS em $28 \%$ da população estudada, sendo, a maioria do sexo masculino (94\%) (9). Martins e colaboradores também observaram prevalência de hipertensão arterial de 23,2\%, sendo significativamente maior no sexo masculino $(28,6 \%)$ do que no feminino (19,7\%) (10). Estes resultados também foram encontrados neste estudo, no qual foi observada uma associação significativa entre o sexo masculino e hipertensão nível I ( $p=0,0061)$. Inquéritos populacionais em cidades brasileiras nos últimos 20 anos apontaram uma prevalência de HAS acima de 30\%. Entre os gêneros, a prevalência foi de 35,8\% nos homens e de $30 \%$ em mulheres, semelhante à de outros países (1). Um estudo sobre DCV em trabalhadores do sexo masculino em uma destilaria, mostrou que a mortalidade por DAC e acidente vascular encefálico (AVE), corresponde a $80 \%$ dos óbitos por DCV, apontando a hipertensão arterial sistêmica (HAS) como fator de risco mais importante para as $\mathrm{DCV}$, sendo $80 \%$ das mortes por acidente vascular encefálico e 40\% dos óbitos por DC resultados de HAS (11). A HAS tem alta prevalência e baixas taxas de controle, é considerada um dos principais fatores de risco modificáveis e um dos mais importantes problemas de saúde pública. A mortalidade por doença cardiovascular (DCV) aumenta progressivamente com a elevação da PA a partir de 115/75 $\mathrm{mmHg}$ de forma linear, contínua e independente (1). 
Tabela 5 - Distribuição das médias e desvios padrão de circunferência de cintura (CC), circunferência de quadril (CQ) e relação circunferência cintura/circunferência do quadril (RCQ) por faixa etária, no sexo masculino.

\begin{tabular}{|c|c|c|c|c|c|}
\hline & $\mathbf{3 0}$ anos & $\mathbf{3 1 -} \mathbf{4 0}$ anos & $\mathbf{4 1 - 5 0}$ anos & $>\mathbf{5 0}$ anos & ANOVA p \\
\hline CC & $90,95 \pm 15,58$ & $93,42 \pm 8,78$ & $104,17 \pm 10,48$ & $94,00 \pm 20,17$ & 0,2586 \\
\hline CQ & $104,58 \pm 13,36$ & $102,92 \pm 6,99$ & $113,83 \pm 12,92$ & $106,6 \pm 6,46$ & 0,2506 \\
\hline RCQ & $0,86 \pm 0,05$ & $0,90 \pm 0,05$ & $0,92 \pm 0,05$ & $0,87 \pm 0,15$ & 0,0980 \\
\hline
\end{tabular}

Tabela 6 - Distribuição das médias e desvios padrão de circunferência de cintura (CC), circunferência de quadril (CQ) e relação circunferência de cintura/circunferência do quadril (RCQ) por faixa etária, no sexo feminino.

\begin{tabular}{|c|c|c|c|c|c|} 
& $\leq \mathbf{3 0}$ anos & $\mathbf{3 1 - 4 0}$ anos & $\mathbf{4 1 - 5 0}$ anos & $>\mathbf{5 0}$ anos & ANOVA P \\
\hline CC & $76,42 \pm 10,71^{*} \dagger$ & $83,92 \pm 13,68$ & $87,82 \pm 15,08$ & $92,70 \pm 16,02$ & 0,003 \\
\hline CQ & $102,45 \pm 8,94$ & $105,23 \pm 9,42$ & $105,41 \pm 9,50$ & $110,5 \pm 10,86$ & 0,1352 \\
\hline RCQ & $0,74 \pm 0,07$ & $0,79 \pm 0,08$ & $0,83 \pm 0,09$ & $0,83 \pm 0,08$ & 0,0561 \\
\hline
\end{tabular}

* Bonferroni: $\mathrm{p}<0.01$ vs 41 a 50 anos;

$\dagger$ Bonferroni: $\mathrm{p}<0.01 \mathrm{vs}>50$ anos

O fato de, neste estudo, a grande maioria dos funcionários $(82,68 \%)$ relatarem história familiar positiva prévia de doenças cardiovasculares é bastante preocupante uma vez que este é um fator de risco bem estabelecido. Em nosso país, a história familiar de doenças cardiovasculares tem sido associada à presença de níveis alterados de colesterol total e risco cardiovascular (12). Um estudo de risco cardiovascular em funcionários de uma indústria encontrou história familiar pregressa de doença arterial coronariana em parentes de primeiro grau em 41 mulheres, correspondendo a $31 \%$ da população feminina, e 184 homens (20\%) (9). Estudos amplos de Associação Genômica nos últimos anos são a prova imparcial de que os genes contribuem para traços comuns de doença cardiovascular em europeus e algumas populações asiáticas. Os resultados confirmam o papel central das lipoproteínas, e revelam que ainda há muito a aprender sobre os mecanismos subjacentes da doença, como a maioria dos genes associados ligados à aterosclerose (13).

O fumo é considerado importante causa na perda da saúde, sendo um hábito bastante frequente na sociedade brasileira. Neste estudo, o tabagismo foi encontrado como hábito em $9,45 \%$ dos funcionários. Resultados semelhantes foram observados em funcionários da Petrobrás, nos quais $69,4 \%$ da população nunca haviam fumado, $18,2 \%$ eram ex-fumantes e apenas $12,4 \%$ tabagistas (14). De acordo com o Ministério da Saúde (5) o hábito de fumar se mostra mais disseminado entre homens do que entre mulheres em todas as cidades do Brasil, o que também foi observado no presente trabalho, no qual $7,14 \%$ das mulheres relataram tabagismo pregresso e $18,6 \%$ dos homens, indicando que, no total, 14 indivíduos $(11,02 \%)$ da população estudada foram expostos a esse fator de risco. Dados do Ministério da Saúde apontam que mais da metade dos homens e cerca de $33 \%$ das mulheres adultas fumam cigarro. Além disso, o fumo é responsável por um quinto das mortes por DCV, e por $29 \%$ da mortalidade por DAC $(11 ; 15)$.

A relação entre atividade física, saúde e qualidade de vida vem sendo cada vez mais discutida e comprovada cientificamente. Neste estudo foi constatado elevado nível de sedentarismo $(66,14 \%)$, com frequência maior no sexo feminino $(73,81 \%)$. Pode-se observar uma associação significativa entre sexo feminino e sedentarismo $(\mathrm{p}=0,0168)$. Um estudo em usuários do Sistema Único de Saúde de Goiânia encontrou uma prevalência de $54,8 \%$ para sedentarismo, quando considerado ausência de atividades físicas que geram gasto energético seja ocupacional no lazer, no trabalho doméstico -, seja como meio de locomoção (16). Atividade física reduz a incidência de HAS, mesmo em indivíduos pré-hipertensos, bem como a mortalidade e o risco de DCV (1).

Neste estudo, aproximadamente $50 \%$ da população relataram etilismo, com prevalência no sexo masculino $(69,77 \%)$, resultando em uma associação altamente significativa entre sexo masculino e consumo de álcool $(\mathrm{p}=0,0014)$. Martins e colaboradores (10) relataram o consumo de bebidas alcoólicas em metade dos participantes do seu estudo, nos trinta dias anteriores à entrevista, independentemente da quantidade consumida. De acordo com o Ministério da Saúde (15), na maioria das cidades, a frequência do consumo abusivo de bebidas alcoólicas é duas 
vezes maior em homens do que em mulheres. A ingestão de álcool por períodos prolongados de tempo pode aumentar a pressão arterial e a mortalidade cardiovascular em geral. Em populações brasileiras o consumo excessivo de etanol se associa com a ocorrência de HAS de forma independente das características demográficas (1). O consumo de bebidas alcoólicas é culturalmente aceito pelas diferentes populações, com significados culturais e simbólicos próprios. O consumo é facilitado pelo baixo custo, tornando a bebida alcoólica acessível a todas as camadas sociais. Porém, o álcool é uma substância capaz de causar além dos problemas sociais como acidentes, violência, absenteísmo e desemprego, os problemas clínicos como a hipertensão arterial, cirrose hepática e outras patologias (17).

$\mathrm{O}$ estresse é considerado um fator de risco cardiovascular independente, e ainda, contribui para o aumento dos demais fatores de risco, podendo ser causa de obesidade, hipertensão arterial, isquemia miocárdica, arritmias cardíacas, aumento dos níveis de colesterol e glicose e até mesmo morte súbita. Os danos físicos e mentais causados não se devem ao número de eventos estressantes experimentados pelo indivíduo, e sim à sua capacidade de lidar com tais eventos (18). No presente estudo, 33,86\% dos indivíduos relataram estresse. Destes, a maior frequência foi relacionada ao sexo feminino $(38,10 \%)$, porém sem diferença significativa. O sistema cardiovascular é reconhecidamente vulnerável ao estresse psicossocial, pois inúmeros fatores psicossociais têm implicados na patogênese da doença arterial coronária (11).

O excesso de peso se associa com maior prevalência de HAS desde idades jovens. Na vida adulta, mesmo entre indivíduos fisicamente ativos, incremento de $2,4 \mathrm{~kg} / \mathrm{m}^{2}$ no índice de massa corporal (IMC) acarreta maior risco de desenvolver hipertensão (1). No presente estudo, com relação ao índice de massa corpórea (IMC), 52,76\% da população total encontra-se nas faixas de pré-obesidade ou obesidade. Houve variação entre os dois sexos, com uma associação significativa entre IMC normal e sexo feminino ( $\mathrm{p}=0,0395)$. No sexo masculino, a maior prevalência foi de pré-obesidade $(41,86)$. Dados semelhantes foram observados em uma análise sobre o diagnóstico do estado nutricional de uma população na região sul do Brasil com prevalência de sobrepeso e obesidade (19). Um estudo retrospectivo em 1213 adultos estabeleceu que o índice de massa corpórea se correlacionou de forma significativa com alto ris- co cardiovascular $(\mathrm{p}<0.0001)(20)$. Uma variação significativa no sexo masculino foi observada, no presente estudo, quando se comparou IMC relacionado ao nível educacional, com maiores valores de IMC no grupo com grau de instrução maior (ANOVA $\mathrm{P}=0,012)$. Este resultado difere do observado por outros estudos que demonstram que indivíduos com melhor grau de instrução apresentam menores IMC, notadamente no sexo masculino (9). O uso do IMC, como uma medida de classificação do estado nutricional pode ser útil em estudos populacionais, embora pouco refinada em relação à distribuição da gordura corporal. Assim, as medidas como a RCQ e a $\mathrm{CC}$ podem dar informação adicional quanto à natureza da obesidade (21). No presente estudo podemos ressaltar a prevalência de alterações na circunferência de cintura, circunferência de quadril e razão cintura/quadril, notadamente no sexo feminino, com incremento significativo de CC com o aumento da idade $(\mathrm{p}=0,003)$. Vários estudos relacionam o IMC e a RCQ como os indicadores antropométricos com maior correlação com o perfil lipídico em ambos os sexos, sustentando a hipótese de que o IMC e a RCQ podem ser considerados como fatores de risco para a doença cardiovascular $(19,22,23)$.

Vale destacar que a obesidade abdominal está vinculada a um maior risco cardiovascular, pelo fato da gordura nessa região depositada estar mais relacionada às alterações no metabolismo de lipídios, e sua identificação caracteriza um paciente com alto risco de desenvolver doenças cardiovasculares (24, 25, 26).

\section{CONCLUSÃO}

Este estudo identificou uma clara prevalência de fatores de risco cardiovascular no grupo analisado, com a ocorrência de vários fatores de risco modificáveis. Conhecer os fatores de risco se traduz em uma posição positiva no que diz respeito à prevenção e reforça a necessidade da implantação de programas educativos de orientação preventiva que incluam a conscientização da população quanto aos benefícios de adoção de um estilo de vida saudável.

\section{AGRADECIMENTOS}

A todos os indivíduos incluídos neste estudo. 


\section{REFERÊNCIAS}

1. Sociedade Brasileira de Cardiologia / Sociedade Brasileira de Hipertensão / Sociedade Brasileira de Nefrologia. VI Diretrizes Brasileiras de Hipertensão. Arq Bras Cardiol 2010; 95(supl.1): $1-51$

2. Muller EV, Aranha SRR, Roza WSS, Gimen SGA. Distribuição espacial da mortalidade por doenças cardiovasculares no Estado do Paraná, Brasil: 1989-1991 e 2006-2008. Cad. Saúde Pública, Rio de Janeiro, 28(6):1067-1077, jun, 2012

3. Nozawa D, Franken RA, Ribeiro KCB, Pereira AC, Sprovieri SRS, Golin V. Estudo comparativo entre pacientes infartados fumantes, ex-fumantes e não-fumantes. Arq. Bras. Cardiol. 2003; 81(6): 589-91

4. Silva RB, Costa-Paiva L, Pinto NAM, Braga AA, Morais SS. Atividade física habitual e risco cardiovascular na pós-menopausa. Rev. Assoc. Med. Bras. 2006; 52(4): 242-246.

5. Sposito AC, Caramelli B, Fonseca FAH, Bertolami MC, Afiune NA, Souza AD, et al. IV Diretriz Brasileira sobre Dislipidemias e Prevenção da Aterosclerose: Departamento de Aterosclerose da Sociedade Brasileira de Cardiologia. Arq. Bras. Cardiol. 2007, 88(suppl.1): 2-19.

6. World Health Organization (WHO). Obesity: preventing and managing the global epidemic. Report of a WHO Consultation (WHO Technical Report Series 894), 2000. Disponível em: http:// www.who.int/nutrition/publications/obesity/WHO TRS 894/ en/index.html

7. Godoy MF, Lucena JM, Miquelin AR, Paiva FF, Oliveira DLQ, Augustin Jr JL, et al. Mortalidade por doenças cardiovasculares e níveis socioeconôomicos na população de São José do Rio Preto, Estado de São Paulo, Brasil. Arq Bras Cardiol. 2007; 88 (2): 2006.

8. Cesarino CB, Cipullo JP, Martin JFV, Ciorlia LA, Godoy MRP, Cordeiro, JÁ, Rodrigues IC. Prevalência e Fatores Sociodemográficos em Hipertensos de São José do Rio Preto - SP. Arq Bras Cardiol. 2008; 91(1): 31-5.

9. Cassani RSL, Nobre F, Pazin FA, Schmidt A. Prevalência de fatores de risco cardiovascular em trabalhadores de uma indústria brasileira. Arq. Bras. Cardiol. 2009; 92(1): 16-22.

10. Martins MSAS, Ferreira MG, Guimarães LV, Vianna LAC. Hipertensão Arterial e Estilo de Vida em Sinop, Município da Amazônia Legal. Arq Bras Cardiol. 2010; 94(5): 639-44.

11. Simão M, Nogueira MS, Hayashida M, Cesarino EJ. Doenças cardiovasculares: perfil de trabalhadores do sexo masculino de uma destilaria do interior paulista. Revista Eletrônica de Enfermagem. 2002; 4(2): 27-35.

12. Mendes GA, Martinez TL, Izar MC, Amancio OM, Novo NF, Matheus SC et al. Perfil lipídico e efeitos da orientação nutricional em adolescentes com história familiar de doença arterial coronariana prematura. Arq. Bras. Cardiol. 2006; 86(5): 361-5.

13. Lusis AJ. Genetics of Atherosclerosis. Trends Genet. 2012, 28(6): 267-275
14. Matos MFD, Silva NAS, Pimenta AJM, Cunha AJLA. Prevalência dos fatores de risco para doença cardiovascular em funcionários do Centro de Pesquisas da Petrobras. Arq. Bras. Cardiol. 2004; 82(1): 1-4.

15. Ministério da Saúde. Principais resultados do Sistema de Monitoramento de Fatores de Risco e Proteção para Doenças Crônicas Não Transmissíveis (VIGITEL). 2007. [citado 2010 dez 2]. Disponível em: http://portal.saude.gov.br/portal/arquivos/pdf/ resumo_relatorio_vigitel_2006_marco_2007.pdf

16. Ferreira CCC, Peixoto MRG, Barbosa MA, Silveira ÉA. Prevalência de fatores de risco cardiovascular em idosos usuários do Sistema Único de Saúde de Goiânia. Arq. Bras. Cardiol. 2010; 95(5): 621-8.

17. Laranjeira R, Romano M. Consenso brasileiro sobre políticas públicas do álcool. Rev Bras Psiquiatr. 2004; 26 (supl 1): S68-77.

18. Heinisch RH, Zukowski CN, Heinisch LMM. Fatores de risco cardiovascular em acadêmicos de medicina. ACM. Arq. Catarin. Med. 2007; 36(1): 76-84.

19. Oliveira MAM, Fagundes RLM, Moreira EAM, Trindade EBSM, Carvalho T. Relação de indicadores antropométricos com fatores de risco para doença cardiovascular. Arq. Bras. Cardiol. 2010; 94(4): 478-85.

20. Cercato C, Mancini MC, Arguell AMC, Passos Q, Villares SMF, Halpern A. Systemic hypertension, diabetes mellitus, and dyslipidemia in relation to body mass index: evaluation of a Brazilian population. Rev Hosp Clin. 2004; 59 (3): 113-8.

21. Rosa MLG, Mesquita ET, Rocha ERR, Fonseca VM. Índice de massa corporal e circunferência da cintura como marcadores de hipertensão arterial em adolescentes. Arq Bras Cardiol. 2007; 88 (5): $573-8$

22. Velasquez-Meléndez G, Kac G, Tavares R, Silva CQ, Garcia ES. Evaluation of waist circumference to predict general obesity and arterial hypertension in women in Greater Metropolitan Belo Horizontal, Brazil. Cad Saúde Pública. 2002; 18 (3): 765-71.

23. Martins MCC, Ricarte IF, Rocha CHL, Maia RB, Silva VB, Veras $\mathrm{AB}$ et al . Pressão arterial, excesso de peso e nível de atividade física em estudantes de universidade pública. Arq. Bras. Cardiol. 2010; 95(2): 192-9

24. Fonseca HAR, Rech CR. 2009. Fatores de risco cardiovascular em mulheres da associação de obesos do município de Ponta Grossa-PR. Arq Ciênc Saúde. 2009; 16(4):170-4.

25. Bergmann GG, Gaya A, Halpern R, Bergmann MLA, Rech RR, Constanzi CB, Alli LR. Circunferência da cintura como instrumento de triagem de fatores de risco para doenças cardiovasculares em escolares. J Pediatr. 2010; 86(5): 411-6.

26. Vasques ACJ, Priore SE, Rosado LEFPL, Franceschini SCC. Utilização de medidas antropométricas para a avaliação do acúmulo de gordura visceral. Rev. Nutr., Campinas. 2010; 23(1): 107-18. 